This is a postprint version of the following published document:

S. Kuru, J. Negro and L Sourrouille. Confinement of Dirac electrons in graphene magnetic quantum dots. In: Journal of Physics: Condensed Matter, 2018, Volume 30, Number 36. DOI: https://doi.org/10.1088/1361-648X/aad656

(C) 2018 IOP Publishing Ltd 


\title{
Confinement of Dirac electrons in graphene magnetic
} quantum dots

\author{
Ş. Kuru ${ }^{a}$, J. Negro $^{b}$ and L. Sourrouille ${ }^{c}$ \\ ${ }^{a}$ Department of Physics, Faculty of Science, Ankara University, 06100 Ankara, Turkey \\ ${ }^{b}$ Departamento de Física Teórica, Atómica y Óptica, Universidad de Valladolid, \\ 47011 Valladolid, Spain \\ ${ }^{c}$ IFISUR-CONICET, B8000CPB Bahía Blanca,/Argentina
}

July 19, 2018

\begin{abstract}
We characterize the confinement of massless Dirac electrons under axially symmetric magnetic fields in graphene, including zero energy modes and higher energy levels. In particular, we analyze in detail the Aharonov-Casher theorem, on the existence of zero modes produced by magnetic fields with finite flux in two dimensions. We apply techniques of supersymmetric quantum mechanics to determine the confined states by means of the quantum number $j$ associated to isospin and angular momentum. We focus on magnetic fields, regular at the origin, whose asymptotic behaviour is $B(r) \sim r^{\alpha}$, with $\alpha$ a real number. A confinement of infinite zero-energy modes and excited states is possible as long as $\alpha \geq-1$. When $-2 \leq \alpha<-1$ the quantum dot is able to trap an infinite number of zero modes but no excited states, while for $\alpha<-2$ only a finite number of zero modes are confined.
\end{abstract}

PACS: $03.65 .-\mathrm{w} ;$ 03.65.Pm; 03.65.Ge

KEYWORDS: graphene; supersymmetric quantum mechanics; magnetic quantum dot; zero mode; confinement.

\section{Introduction}

In the continuous limit of low energy electrons in graphene, the stationary states are described by a massless Dirac-Weyl equation in two space dimensions $[1,2]$. The study of the influence of magnetic fields on such quasi-particles has a great interest both from fundamental and applied points of views. Let us just mention, for example, the Landau 
levels and Hall effect or the design of different confining structures $[3,4,5,6]$. The electric fields have a secondary role to produce confinement due to the strong Klein tunnelling characteristic of zero mass particles $[7,8,9,10]$. This difficulty has given rise to consider pure magnetic traps $[11,12]$. Many kinds of magnetic confinement have been treated, for instance with inhomogeneous field profiles $[13,14,15,16,17,18,19,20]$, magnetic antidots [3] or anti-rings [21, 22]. It was shown in [4] that a circular step magnetic field profile leads to a finite number of zero energy modes, but no stable excited energy level is allowed. Nevertheless, recently it was reported that magnetic traps in the form of dots and rings were able to confine excited bound states $[5,6]$. These traps have magnetic fields which are of a slowly decaying nature. Different experimental methods in graphene have been developed to measure the effects of magnetic fields (see $[23,24,25]$ and references therein). Finally, let us mention that other new confining mechanism has been considered by using velocity barriers, where the Fermi velocity differs in different regions [26]. A list of some solvable cases of this type of confining has been given in [27].

The motion of the massless Dirac electrons on the graphene surface is sensitive to perpendicular magnetic fields. In this letter, we want to discuss the confining properties of perpendicular fields with axial symmetry under general conditions. We will pay attention to the zero energy level and its degeneracy as well as to the existence of other bound states. Firstly, we want to apply the Atiyah-Singer index theorem following the arguments of Aharonov and Casher to the case of finite flux magnetic fields with finite support $[28,29,30]$ about zero modes, but as we will see later, in this context other excited states are excluded. Afterwards, we will consider general axially symmetric magnetic fields whose asymptotic behaviour is $B(r) \sim r^{\alpha}$, with $\alpha$ an arbitrary real number, where not only zero modes, but also excited energy levels can exist. We show that a full confinement of infinite zero-energy modes and infinite excited bound states in magnetic quantum dots are indeed possible as long as $\alpha \geq-1$. We also find that if $-2 \leq \alpha<-1$, it is possible to trap only massless Dirac fermions at zero energy with infinite degeneracy. Finally, we explore the case $\alpha<-2$ showing that only a finite number of zero-modes are allowed.

This type of magnetic interacting systems in graphene [16] as well as in fullerenes and nanotubes $[31,32]$ has the formal structure of supersymmetric quantum mechanics (susy-qm) [33, 34, 35, 36]. The symmetry of the field and the supersymmetric algebraic structure allow us to get simple and clear expressions for different properties of these magnetic systems. In particular, by using this framework, we will interpret the zero mode degeneracy in terms of the quantum numbers $j$ and isospin. We will as well examine, for each value of $j$, what type of magnetic fields will allow for the existence of other excited states.

The paper is structured as follows. In Section 2 the notation for an axially symmetric magnetic field, its potentials and gauge freedom is introduced. In Section 3 the formulation of two dimensional Dirac-Weyl equation for two-component spinors in polar coordinates is applied to describe the interaction with these fields. The formal structure of this equation in terms of the susy-qm language is shown together with the effective equations for each 
spinor component. The simplest application to the zero modes of magnetic fields with compact support is given in Section 4. The following section contains another application to a more general class of magnetic quantum dots, which includes many of the most usual cases. The final section is devoted to comment the main results found here.

\section{Axially symmetric magnetic fields in two dimensions}

In the $x$ - $y$ plane, we consider a perpendicular magnetic field $B(r) \hat{z}$ with axial symmetry, generated by the magnetic vector potential $\mathbf{A}=f(r)(-y / r, x / r)$, which can be rewritten in polar coordinates as

$$
\mathbf{A}=f(r) \hat{\theta}, \quad f(r) \equiv \frac{A_{\theta}(r)}{r}
$$

where $\hat{\theta}$ is the unit vector in the azimuthal direction. It is/evident that this vector potential is in the Coulomb gauge, i.e. $\nabla \cdot \mathbf{A}=\frac{1}{r} \partial_{\theta} f(r)=0$, thus it is possible to introduce a scalar field $\phi(r)$ such that

$$
f(r)=\partial_{r} \phi(r)
$$

Here, we use the notation $\partial_{r}=\frac{\partial}{\partial r}$, etc. In terms of the function $f(r)$ (or the field $\phi(r)$ ), the magnetic field $B(r)$ is given by

$$
B(r)=\partial_{r} f(r)+\frac{f(r)}{r}=\Delta \phi(r),
$$

where $\Delta$ is the Laplace operator in two dimensions. This implies that the potentials $f(r)$ and $\phi(r)$ can immediately be obtained from $B(r)$ as follows,

$$
f(r)=\frac{1}{r} \int r B(r) d r+\frac{c_{0}}{r}, \quad \phi(r)=\int \frac{1}{r}\left(\int r B d r\right) d r+c_{0} \log r+c_{1},
$$

where $c_{0}$ and $c_{1}$ are arbitrary integration constants. So, $f(r)$ is defined up to the function $c_{0} / r$, while $\phi(r)$ includes the term $c_{0} \log r+c_{1}$ corresponding to the homogeneous solutions of (2.3). It is worth to notice that, from (2.4), the expression for $A_{\theta}(r)$ takes the form

$$
A_{\theta}(r)=\int r B(r) d r+c_{0}
$$

with the constant $c_{0}$ associated to the remaining gauge freedom. We can fix it by choosing the lower limit of the integral at the origin, provided $B$ is not highly singular there. Then, $A_{\theta}(r)$ will be given by the flux $\Phi(r)$ of the field $B(r)$ in the disk of radius $r$,

$$
A_{\theta}(r)=\frac{1}{2 \pi} \Phi(r), \quad \Phi(r)=2 \pi \int_{0}^{r} r^{\prime} B\left(r^{\prime}\right) d r^{\prime}
$$

In Table 1, we show some simple cases of magnetic fields with the associated functions $f(r)$ and $\phi(r)$. 


\begin{tabular}{|c|c|c|}
\hline$B(r)$ & $f(r)$ & $\phi(r)$ \\
\hline 0 & $\frac{c_{0}}{r}$ & $c_{0} \log r+c_{1}$ \\
\hline$b_{0}$ & $\frac{b_{0}}{2} r$ & $\frac{b_{0}}{4} r^{2}$ \\
\hline$\frac{b_{0}}{r}$ & $b_{0}$ & $b_{0} r$ \\
\hline$b_{0} r$ & $\frac{b_{0}}{3} r^{2}$ & $\frac{b_{0}}{9} r^{3}$ \\
\hline
\end{tabular}

Table 1: Examples of magnetic fields $B(r)$ together with its potentials $f(r)=A_{\theta}(r) / r$ and $\phi(r)$. The functions $f(r)$ and $\phi(r)$ are non unique, as mentioned in the text.

\section{External symmetric magnetic fields in graphene}

Graphene consists of a two-dimensional periodic hexagonal honeycomb array of carbon atoms. The tight-binding Hamiltonian of graphene in the vicinity of one of the two independent Dirac points of the hexagonal Brillouin zone, where the energy goes to zero, reduces to the two-dimensional massless Dirac-Weyl Hamiltonian $[1,2]$

$$
H \Psi=\left(\sigma_{x} p_{x}+\sigma_{y} p_{y}\right) \Psi=E \Psi
$$

where $E=\mathcal{E} / v_{F}, \mathcal{E}$ is the energy and $v_{F}$ the Fermi velocity; in addition, $p_{j}=-i \hbar \partial_{j}$ $(j=x, y)$ represents the two-dimensional momentum operator and $\sigma_{i}(i=x, y)$ the Pauli matrices [1]. The interaction of the electrons of charge $q$ with an external magnetic field is described via the minimal coupling rule through the magnetic potential $\mathbf{A}$ (we will take $c=\hbar=1$,

$$
H \Psi=\left(\sigma_{x}\left(p_{x}-q A_{x}\right)+\sigma_{y}\left(p_{y}-q A_{y}\right)\right) \Psi=E \Psi .
$$

In our case, due to the axial symmetry, we write this Hamiltonian in polar coordinates $(x, y)=(r \cos \theta, r \sin \theta)$, so that the expression of $H$ in (3.2) becomes

$$
H=\left(\begin{array}{cc}
0 & i e^{-i \theta}\left(-\partial_{r}+\frac{i \partial_{\theta}}{r}+q f(r)\right) \\
-i e^{i \theta}\left(\partial_{r}+\frac{i \partial_{\theta}}{r}+q f(r)\right) & 0
\end{array}\right)
$$

where we have taken into account the notation (2.1) for the magnetic vector potential. We will also use the following notation for the first order differential operators appearing in 
the matrix Hamiltonian (3.3),

$$
A^{ \pm}=e^{\mp i \theta}\left(\mp \partial_{r}+\frac{i \partial_{\theta}}{r}+q f(r)\right)=e^{\mp i \theta}\left(\mp \partial_{r}+\frac{i \partial_{\theta}+q A_{\theta}(r)}{r}\right)
$$

In this expression it is explicit the minimal coupling and the remaining gauge invariance is implemented by the transformations:

$$
A_{\theta}(r) \rightarrow A_{\theta}(r)+c_{0}, \quad \Psi \rightarrow e^{i q c_{0} \theta} \Psi .
$$

However, this can only be realized as far as $q c_{0}=k$, with $k \in \mathbb{Z}$, because $\Psi$ must be single valued. Therefore, the clases of inequivalent potentials can be parametrized, for instance, by $q c_{0} \in[0,1)$ (or by any other interval of a unit length). Taking in mind these considerations, $H$ can be rewritten as

$$
H=\left(\begin{array}{cc}
0 & i A^{+} \\
-i A^{-} & 0
\end{array}\right)
$$

Since our system is symmetric under rotation around the $z$-axis, the "total angular momentum" $J_{z}$ is conserved, so that we can look for energy eigenfunctions that at the same time are eigenfunctions of $J_{z}$ :

$$
J_{z} \Psi=\left(L_{z}+\frac{1}{2} \sigma_{z}\right) \Psi=j \Psi .
$$

As the orbital angular momentum is $L_{z}=-i \partial_{\theta}$, the wave functions $\Psi$ with eigenvalue $j=\ell+1 / 2$ will take the form

$$
\Psi(r, \theta)=\left(\begin{array}{c}
\psi_{1}(r, \theta) \\
\psi_{2}(r, \theta)
\end{array}\right)=\left(\begin{array}{c}
\xi(r) e^{i \ell \theta} \\
-i \chi(r) e^{i(\ell+1) \theta}
\end{array}\right)
$$

where $\ell$ is for the integer eigenvalues of $L_{z}$, and the subindex 1,2 of the spinor components refer to the sublattices $A, B$, respectively, of the graphene honeycomb lattice. Then, the equations to be satisfied by the radial components $\xi(r)$ and $\chi(r)$ of an eigenfunction $\Psi$, with $j=\ell+1 / 2$, according to the above notation, are

with

$$
A_{\ell+1}^{+} \chi(r)=E \xi(r), \quad A_{\ell}^{-} \xi(r)=E \chi(r)
$$

Therefore, the equations for each component have the form

$$
H_{1} \xi(r) \equiv A_{\ell+1}^{+} A_{\ell}^{-} \xi(r)=E^{2} \xi(r), \quad H_{2} \chi(r) \equiv A_{\ell}^{-} A_{\ell+1}^{+} \chi(r)=E^{2} \chi(r) .
$$


In these expressions, $H_{1}$ and $H_{2}$ are second order differential operators in $r$ that include first order derivatives. In order to get rid of them, we perform the transformation

$$
\xi=r^{-1 / 2} \tilde{\xi}, \quad \chi=r^{-1 / 2} \tilde{\chi} .
$$

In this way, the equations (3.11) come into

$$
\tilde{H}_{1} \tilde{\xi}(r)=\tilde{A}_{j}^{+} \tilde{A}_{j}^{-} \tilde{\xi}(r)=E^{2} \tilde{\xi}(r), \quad \tilde{H}_{2} \tilde{\chi}(r)=\tilde{A}_{j}^{-} \tilde{A}_{j}^{+} \tilde{\chi}(r)=E^{2} \tilde{\chi}(r),
$$

with

$$
\tilde{A}_{j}^{ \pm}=\mp \partial_{r}-\frac{j}{r}+q f(r), \quad j=\ell+1 / 2, \quad \ell \in \mathbb{Z} .
$$

The expressions (3.13)-(3.14), including the "good" quantum number $j$, are the basic ingredients of the susy-qm structure of the effective partner Hamiltonians $\tilde{H}_{1}$ and $\tilde{H}_{2}$ corresponding to $j$ total momentum, with intertwining operators $\tilde{A}_{j}^{ \pm}$. The Hamiltonians $\tilde{H}_{1,2}$ have effective potentials defined by

$$
\tilde{H}_{1,2}=-\partial_{r r}+\tilde{V}_{1,2}(r)
$$

with

$$
\tilde{V}_{1,2}(r)=W(r)^{2} \pm \frac{d W(r)}{d r},
$$

where the superpotential $W(r)$ has the form

$$
W(r)=\frac{j}{r}-q f(r)=\frac{j-q A_{\theta}(r)}{r} .
$$

The gauge transformation (3.5) is realized by the change $A_{\theta}(r) \rightarrow A_{\theta}(r)+c_{0}$ and $j \rightarrow j+q c_{0}$, with $q c_{0} \in \mathbb{Z}$ in (3.17), so that this expression remains invariant. However, the change in the potential $A_{\theta}(r)$ given, for instance, by the constants $q c_{0} \in(0,1)$ correspond to inequivalent gauge classes of potentials for the same magnetic field that can not be compensated by a change of $j$ (which takes only discrete half odd values).

\section{Zero modes and excited states for a field $B(r)$ of compact support}

Next, we will characterize the zero modes of the Hamiltonian (3.3). According to the relations (3.9) of the components of the eigenfunctions, such zero modes are given by one of the following expressions

$$
\tilde{\Psi}_{0}^{+}=\left(\begin{array}{c}
\tilde{\xi}_{0} \\
0
\end{array}\right), \quad \tilde{\Psi}_{0}^{-}=\left(\begin{array}{c}
0 \\
\tilde{\chi}_{0}
\end{array}\right)
$$


where the respective components $\tilde{\xi}_{0}$ or $\tilde{\chi}_{0}$ must satisfy

$$
\tilde{A}_{j}^{-} \tilde{\xi}_{0}=0, \quad \tilde{A}_{j}^{+} \tilde{\chi}_{0}=0 .
$$

The zero modes are characterized by the total momentum number $j$, but also by the isospin $S_{z} \tilde{\Psi}_{0}^{ \pm}=\frac{1}{2} \sigma_{z} \tilde{\Psi}_{0}^{ \pm}= \pm \frac{1}{2} \tilde{\Psi}_{0}^{ \pm}$. The explicit expressions for such components are (up to a normalization constant)

$$
\tilde{\xi}_{0}(r)=r^{j} e^{-q \int_{0}^{r} f(r) d r}, \quad \tilde{\chi}_{0}(r)=r^{-j} e^{q \int_{0}^{r} f(r) d r}
$$

A physical zero mode wavefunction must have square integrable components, so it is given by a non vanishing $\tilde{\xi}_{0}(r)$ or by $\tilde{\chi}_{0}(r)$, but not by both. This character is determined by the behaviour of $f(r)$. We can check that the function $W(r)$ in (3.17) can also be computed from a zero energy eigenfunction $\tilde{\xi}_{0}(r)$ (or $\left.\tilde{\chi}_{0}(r)\right)$ of $\tilde{H}_{1}\left(\tilde{H}_{2}\right)$ :

$$
W(r)=\frac{\partial_{r} \tilde{\xi}_{0}(r)}{\tilde{\xi}_{0}(r)}=-\frac{\partial_{r} \tilde{\chi}_{0}(r)}{\tilde{\chi}_{0}(r)} .
$$

In fact, a result of susy-qm says that the potential $\tilde{V}_{1}(r)$ can be obtained through $W(r)$ defined as in (4.4) by means of any zero-energy wayefunction $\tilde{\xi}_{0}(r)$ of $\tilde{H}_{1}$, even if it is not square integrable.

Now, assume that the magnetic field has a compact support, determined by the disk of radius $r_{0}>0$,

$$
B(r)= \begin{cases}b(r), & r<r_{0} \\ 0, & r \geq r_{0}\end{cases}
$$

where $b(r)$ is a smooth function. Let us consider first the case where the total flux $\Phi_{0}$ be finite and positive. Then, taking into account (2.6), the component $A_{\theta}$ is constant outside the disk:

hence from $(2.1)$,

$$
A_{\theta}(r)=\frac{1}{2 \pi} \Phi_{0}, \quad r>r_{0},
$$

In this case, the square integrable zero modes are of $\xi$-type

$$
\tilde{\xi}_{0}(r)=r^{j} r^{-\frac{q}{2 \pi} \Phi_{0}}, \quad r>r_{0}
$$

and, therefore $j$ must be bounded from above: $j<\frac{q}{2 \pi} \Phi_{0}-\frac{1}{2}, j=1 / 2, \ldots$ On the other hand, in order $\tilde{\xi}_{0}(r)$, as given by (4.3), be regular at the origin, the values of $j$ must also be bounded from below: $1 / 2 \leq j$. Thus, the number of zero modes will be given by the finite values $j$ such that

$$
\frac{1}{2} \leq j<\frac{q}{2 \pi} \Phi_{0}-\frac{1}{2}, \quad j=1 / 2,3 / 2, \ldots j_{\max }
$$


where $j_{\max }$ is the maximum half odd satisfying the inequality (4.9). Similar considerations apply when the field $B(r)$ is of compact support and with finite negative total flux $\Phi_{0}<0$. Then, the zero modes will be of $\chi$-type and negative $j$-momentum,

$$
\tilde{\chi}_{0}(r)=r^{-j} r^{\frac{q}{2 \pi} \Phi_{0}}, \quad r>r_{0}
$$

where

$$
-\frac{1}{2} \geq j>\frac{q}{2 \pi} \Phi_{0}+\frac{1}{2}, \quad j=-1 / 2,-3 / 2 \ldots j_{\min } .
$$

This is essentially the result of Aharonov-Casher [28] for the specific case of axially symmetric fields. There are three points worth to be remarked.

(i) It is possible that there could be no zero modes at all, this happens if the total flux is small enough: $\left|\frac{q}{2 \pi} \Phi_{0}\right| \leq 1$.

(ii) In the formulas for the number of zero modes of a magnetic field $B(r)$, we have selected the gauge of the potential, given in (2.6), such that $A_{\theta}(r)=\frac{1}{2 \pi} \Phi(r)$, where $\Phi(r)$ is the flux of the magnetic field. However, we can fix now the inequivalent classes of potentials in the following way. Take $j_{\max }$ in the case where formula (4.9) apply: $j_{\max }<\frac{q}{2 \pi} \Phi_{0}-\frac{1}{2}$. Then we can choose the unit interval for the constant $q c_{0}$ of gauge potentials such that

$$
\frac{q}{2 \pi} \Phi_{0}-\frac{1}{2}+q c_{0} \in\left(j_{\max }, j_{\max }+1\right] .
$$

This choice implies that all the inequivalent gauge potentials for a magnetic field $B(r)$ of compact support have the same number of zero modes. This seems the "natural choice" for the parametrization of gauge potentials for magnetic fields with finite flux.

(iii) Assume that there exist a finite number of zero modes (or even no zero modes), there can exist other excited states? We will show next that the answer to this question is negative. The argument is as follows. Assume that the magnetic field fulfils the first type of conditions with a total positive and finite flux $0<\Phi_{0}<+\infty$. Then, a zero energy wavefunction corresponding to any value $j$ of the total momentum, according to (4.8), will have the asymptotic form

$$
\tilde{\xi}(r)=c r^{j_{\mathrm{eff}}}, \quad r>r_{0}, \quad j_{\mathrm{eff}}=j-\frac{e}{2 \pi} \Phi_{0} .
$$

This wavefunction might not be square integrable. Next, if we apply formulas (3.16) and (4.4) to get the effective potential $\tilde{V}_{1}$, we will find

$$
\tilde{V}_{1}(r)=\frac{j_{\mathrm{eff}}\left(j_{\mathrm{eff}}-1\right)}{r^{2}}, \quad r>r_{0}
$$



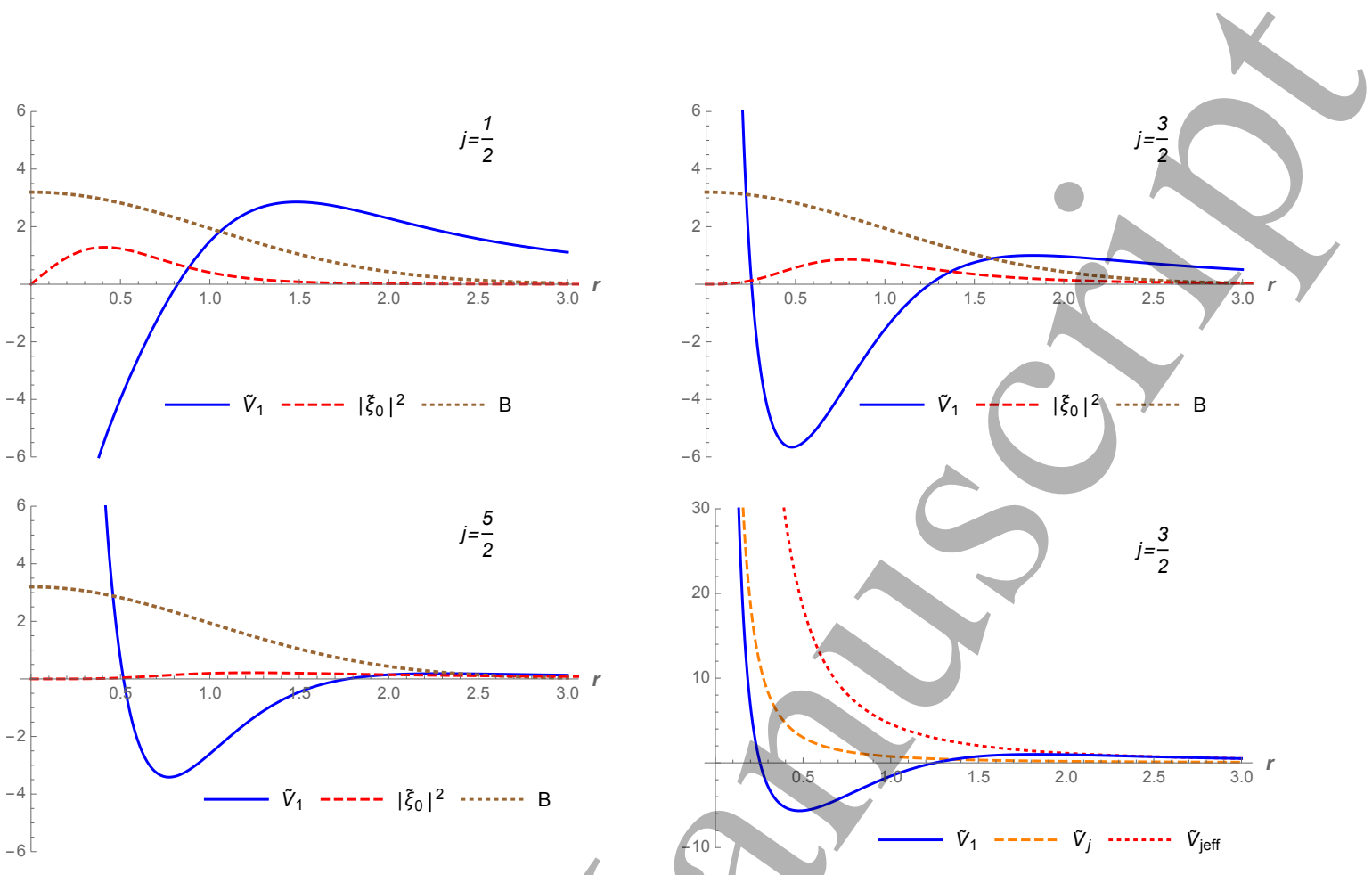

Figure 1: Plot of the effective potentials $\tilde{V}_{1}(r)$ for the magnetic quantum dot $B(r)=3.2 e^{-\frac{r^{2}}{2}}$. There are three zero modes with $j=1 / 2,3 / 2$ (upper left and right) and $j=5 / 2$ (lower left figure). Together with the potential (continuous curve) it is shown the corresponding probability density $\left|\tilde{\xi}_{0}\right|^{2}$ (dashed curve) and the magnetic field (dotted line). In the lower right figure, together with the effective potential $\tilde{V}_{1}$ (continuous curve) for $j=3 / 2$, it is shown the free potential $\tilde{V}_{j}(r)=$ $\frac{j(j-1)}{r^{2}}$ near the origin (dashed line) and the free potential outside the disk of non-vanishing field $\tilde{V}_{j_{\text {eff }}}(r)=\frac{j_{\text {eff }}\left(j_{\text {eff }}-1\right)}{r^{2}}$ (dotted line). The curve of $\tilde{V}_{1}(r)$ interpolates between $\tilde{V}_{j}(r)$ and $\tilde{V}_{j_{\text {eff }}}(r)$, where $\frac{q}{2 \pi} \Phi_{0}=3.2$.

This means that in the limit $r \rightarrow+\infty$ the behaviour of the potential is $\tilde{V}_{1}(r) \rightarrow 0$. In these conditions there can not be an energy $E^{2}>0$ of $\tilde{H}_{1}$ corresponding to bound states since by a tunnelling process such an state would pass to the unbounded region $r>>r_{0}$ where $E^{2}>\tilde{V}_{1}(r)$. In this region, the component $\tilde{\xi}$ will be a linear combination of Bessel functions which can not be normalizable. A similar situation happens with the effective potential $\tilde{V}_{2}(r)$, therefore none of them can support excited states in the presence of finite flux magnetic fields. An illustration of this situation, for the magnetic field $B(r)=3.2 e^{-r^{2} / 2}$, is shown in Fig. 1 . 


\section{General magnetic quantum dots}

We will consider in this section the more general case of axially symmetric quantum dots where the magnetic field is a regular (or regularized) function $b(r)$ in the interior of a disk of radius $r_{0}$, and outside behaving as $r^{\alpha}, \alpha \in \mathbb{R}$ :

$$
B(r)=\left\{\begin{array}{ll}
b(r), & r<r_{0} \\
r^{\alpha}, & r>r_{0}
\end{array} .\right.
$$

\subsection{Zero energy level}

We want to examine the degeneracy of the zero energy level, as well as if there can be excited states, depending on the value of the exponent $\alpha$. According to the magnetic flux formula (2.6),

$$
\Phi(r)=2 \pi \int_{0}^{r_{0}} r b(r) d r+2 \pi \int_{r_{0}}^{r} r^{\alpha+1} d r=\Phi\left(r_{0}\right)+2 \pi \frac{r^{\alpha+2}-r_{0}^{\alpha+2}}{\alpha+2}, \quad r>r_{0},
$$

where the first term $\Phi\left(r_{0}\right)$ is for the magnetic flux inside the disk and the second term is the contribution from the exterior. Now, the flux can have two asymptotic expressions depending on $\alpha$ :

$$
\begin{array}{ll}
\text { (i) } \quad \alpha>-2, & \Phi(r) \sim 2 \pi \frac{r^{\alpha+2}}{\alpha+2}, \quad r>>r_{0}, \\
\text { (ii) } \quad \alpha<-2, & \Phi(r) \sim \Phi_{\text {tot }}, \quad r>>r_{0} .
\end{array}
$$

In case (i) the flux diverges as $r \rightarrow+\infty$, while in case (ii) the total flux $\Phi_{\text {tot }}$ is finite. Hereafter, we assume that in both cases the flux is positive. In this situation, the square integrable zero mode wavefunctions for each of these cases are of type $\xi$ (see (4.1)) and they have the following asymptotic expression in the region $r>>r_{0}$,

$$
\begin{array}{llll}
\text { (i) } \alpha>-2, & \tilde{\xi}_{0}(r) \sim r^{j} e^{-q \frac{r^{\alpha+2}}{(\alpha+2)^{2}}}, & r>>r_{0}, \\
\text { (ii) } \alpha<-2, & \tilde{\xi}_{0}(r) \sim r^{j}\left(\frac{r_{0}}{r}\right)^{\frac{q}{2 \pi} \Phi_{\mathrm{tot}}}, & & r>>r_{0} .
\end{array}
$$

It is clear that for case (i) there are an infinite number of square integrable zero mode states labeled by $j, j=1 / 2,3 / 2 \ldots$ In the second case (ii) where the total flux is finite, the number of zero modes is finite and it is given by a similar condition on $j$ as that of the previous section: $1 / 2 \leq j<\frac{q}{2 \pi} \Phi_{\text {tot }}-1 / 2$.

There is an exceptional case $\alpha=-2$ where the flux is

$$
\Phi(r)=\Phi\left(r_{0}\right)+2 \pi \log \left(r / r_{0}\right), \quad r>r_{0}
$$



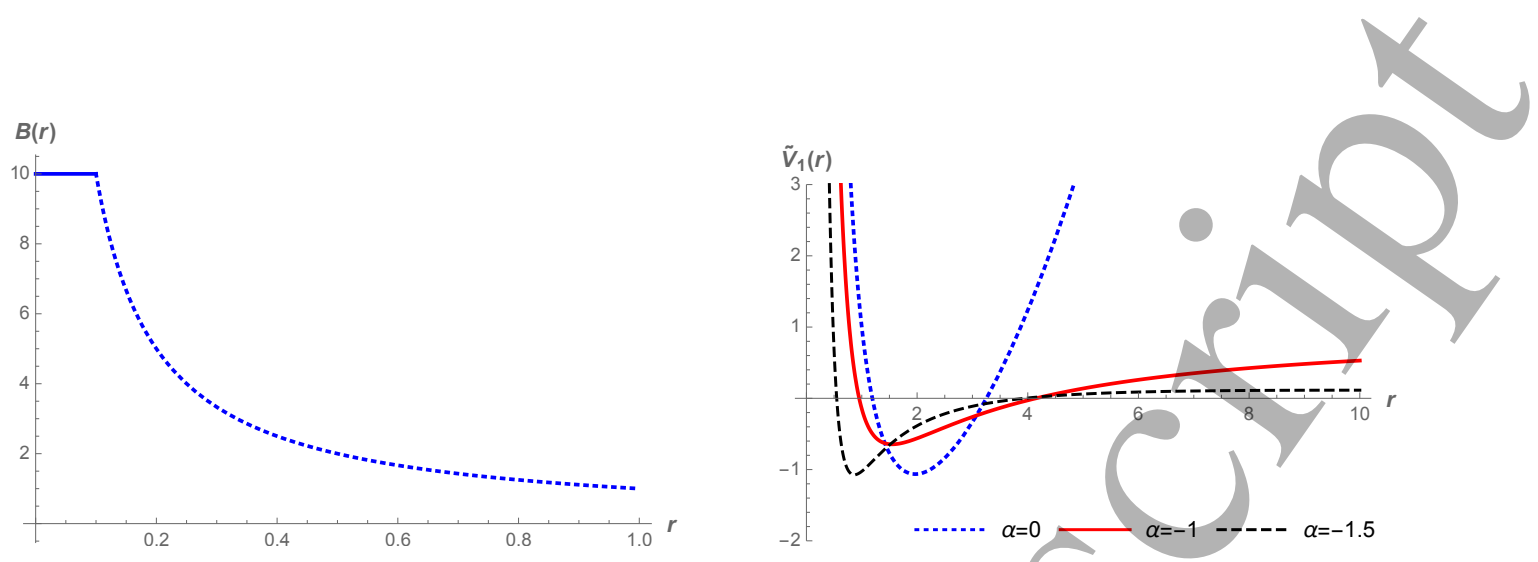

Figure 2: Plot of the magnetic field $B(r)$ (5.8) for $\alpha=-1$ and $r_{0}=0.1$ (at the left). Plot of the effective potentials $\tilde{V}_{1}(r)$ corresponding to this magnetic field for different values of $\alpha$ and $r_{0}=0.1$ (at the right).

The corresponding ground state wave functions, according to (4.3) is

$$
\tilde{\xi}_{0}(r) \propto r^{j} r^{-\frac{q}{2 \pi} \Phi\left(r_{0}\right)} e^{-\frac{q}{2}\left(\log r / r_{0}\right)^{2}}, \quad r>r_{0} .
$$

Therefore, as $\tilde{\xi}_{0}(r)$ is square integrable for all $j>0$, then there will be an infinite number of ground states.

In the limit where $b(r) \rightarrow 0$ in (5.1), we have two cases. For $\alpha \geq-2$, there are no consequences at all, however for $\alpha<-2$ the flux $\Phi_{0} \rightarrow 0$ and there will be no bound states.

\section{$5.2 \quad$ Excited states}

Next, we turn to the problem of the existence of excited states. The best way to get this information is by computing the effective potentials $\tilde{V}_{1}(r)$ corresponding to the asymptotic form of the zero modes given by (5.4) for $\alpha>-2$ by means of the formulas (3.16) and (4.4). We have the following possibilities in case (i) for the dominant term of the asymptotic behaviour of the potential:

$$
\tilde{V}_{1}(r) \sim \frac{q^{2}}{(\alpha+2)^{2}} r^{2(\alpha+1)} \Longrightarrow\left\{\begin{array}{ccc}
(i . a) & \alpha>-1, & \tilde{V}_{1}(r) \underset{r \rightarrow+\infty}{\longrightarrow}+\infty \\
(i . b) & \alpha=-1, & \tilde{V}_{1}(r) \underset{r \rightarrow+\infty}{\longrightarrow} q^{2}>0 \\
(i . c) & -2<\alpha<-1, & \tilde{V}_{1}(r) \underset{r \rightarrow+\infty}{\longrightarrow} 0
\end{array}\right.
$$

Therefore, if $\alpha>-1$, subcase (i.a), the potential well is infinite and there will be an infinite number of bound excited states with discrete spectrum $E_{n}^{2} \rightarrow \infty$. If $\alpha=-1$, the potential well is finite at the right hand side, then we have a similar situation to the Coulomb problem and the number of excited states is also infinite [37,38], but the spectrum is restricted to 
a bounded interval: $0 \leq E_{n}^{2}<q^{2}$. In the last subcase (i.c) when $-2<\alpha<-1$, the right limit of the effective potential vanishes, and there can not be excited states.

As an example, we have taken the following magnetic field (see Fig. 2),

$$
B(r)=\left\{\begin{array}{ll}
r_{0}^{\alpha}, & r<r_{0} \\
r^{\alpha}, & r>r_{0}
\end{array} .\right.
$$

In this case the flux is

$$
\Phi(r)= \begin{cases}2 \pi r_{0}^{\alpha+1}+2 \pi \frac{r^{\alpha+2}-r_{0}^{\alpha+2}}{\alpha+2}, & r>r_{0}, \\ \pi r_{0}^{\alpha} r^{2}, & r<r_{0} .\end{cases}
$$

Then, the superpotential is obtained by (3.17) and the effective potential by (3.16). We have represented different effective potentials $\tilde{V}_{1}(r)$ for the values $\alpha=0,-1,-3 / 2$ and $r_{0}=0.1$ in Fig. 2.

In case (ii), $\alpha<-2$, the asymptotic form of the effective potential takes the form

$$
\tilde{V}_{1}(r) \sim \frac{\left(j-\frac{q}{2 \pi} \Phi_{\mathrm{tot}}\right)\left(j-\frac{q}{2 \pi} \Phi_{\mathrm{tot}}-1\right)}{r^{2}}=\frac{j_{\mathrm{eff}}\left(j_{\mathrm{eff}}-1\right)}{r^{2}}, \quad r>>r_{0} .
$$

We have seen this expression before in (4.14) for the case of fields with finite support. Thus, the potential vanishes in limit in $r \rightarrow+\infty$ and consequently the existence of bound excited states is forbidden.

For the exceptional case $\alpha=-2$, the form of the potential is cumbersome, but from its asymptotic behaviour we conclude that there is no excited states as in the subcase (i.c) corresponding to the values $-2<\alpha<1$.

A summary of the results of this section is contained in Table 2.

\section{Conclusions}

We have studied in detail the zero modes and excited energy levels of graphene under axially symmetric magnetic fields, for low energies near a Dirac $K$-point in the continuum limit.

If the support of the magnetic field is compact and the total flux is finite there is a finite number of zero modes in agreement with the general result obtained by Aharonov-Casher [28] and the Atiyah-Singer theorem [29]. In our specific situation, we have shown that these zero modes correspond to the values of the total angular momentum $j$. If the flux is positive, then the values of $j=\ell+1 / 2$ range from $j=1 / 2$ up to $j_{\max }=\ell_{\max }+1 / 2$. Besides, the spinor zero modes have isospin $1 / 2$ (and therefore, it has only an upper component $\xi$ ) and angular momentum $0 \leq \ell \leq \ell_{\max }$. Thus, only type $A$ atoms in the graphene honeycomb periodic structure contribute to the stationary states. However, if we consider the other 
non-equivalent Dirac $K^{\prime}$-point, the zero modes take negative values from $j=-1 / 2$ up to $j_{\min }=\ell_{\min }-1 / 2$, where $j_{\min }=-j_{\max }$. Here, the isospin is $-1 / 2$ and the spinor has only the lower component $\chi$. Therefore, the stationary zero mode states near the $K^{\prime}$-point come from type $B$ atoms, and the symmetry between $A$ and $B$ atoms of the graphene lattice is restored. For negative magnetic flux the role of the upper and lower components is reversed.

We have shown, in Section 4, that for magnetic fields with finite support and finite total flux, besides the zero modes there are no excited states at all. Any state with positive (or negative) energy is unstable. Outside the support region of the magnetic field, the effective potential for each component of the spinor drops as $j_{\text {eff }}\left(j_{\text {eff }}-1\right) / r^{2}$ which implies that no positive energy can have bounded states. The value (4.13) of the 'effective angular momentum' $j_{\text {eff }}=j-\frac{q}{2 \pi} \Phi_{0}$ in the asymptotic region $r>>r_{0}$ depends on the total angular momentum $j$ felt near the origin minus the contribution coming the total magnetic flux (see the lower right graphic of Fig. 1). In fact, even the zero energy modes can become unstable by any small energy excitation, in particular the modes with higher momentum $j$ are easier to make unstable, as it is shown by their wavefunctions in Fig. 1. The same arguments can be used to show that for general magnetic fields of compact support and finite flux there are no excited bound states, which would complement the Aharonov-Casher result [28].

Only infinite flux magnetic fields can give rise to an infinite number of zero modes, and in this case the excited states may exist. We have shown how this mechanism is realized for a wide class of magnetic quantum dots (including rings and anti-dots) having asymptotic form $B(r)=r^{\alpha}, \alpha \in \mathbb{R}$, in Section 5. For instance, the simplest case is the Landau system of a constant magnetic field $B(r)=b_{0}$, corresponding to $\alpha=0$ (see Fig. 2), with an infinite number of zero modes with $j=1 / 2,3 / 2,5 / 2 \ldots$, (for positive magnetic flux). In 
this case, there are infinite excited energy labels $E_{n}$, not bounded from above, and due to an additional symmetry, each one with infinite degeneracy (but also including states with negative total angular momentum). Another solvable situation with infinite flux is the singular magnetic field $B(r)=b_{0} / r$, for $\alpha=-1$. In this case the singularity at the origin can be regularized keeping the asymptotic form at $r \rightarrow+\infty$ [5]. However other infinite flux magnetic fields, such as $B(r)=b_{0} r^{-3 / 2}, r>r_{0}$, do not allow excited states (Fig. 2), although they allow for an infinite number of zero modes.

Finally, let us mention that if the two dimensional Dirac Hamiltonian (3.2) includes an additional non null mass term

$$
H \Psi=\left(\sigma_{x}\left(p_{x}-e A_{x}\right)+\sigma_{y}\left(p_{y}-A_{y}\right)+m \sigma_{z}\right) \Psi=E \Psi
$$

the considerations concerning the zero modes remain, but for positive magnetic flux the $\xi$ component of zero mode spinors have energy $E=+m$, while for negative flux, the non zero component is $\chi$ and the energy $E=-m(c=1)$. The excited energy levels satisfy $E_{n}^{2}>m^{2}$, provided $\alpha \geq-1$.

\section{Acknowledgments}

This work was partially supported by the Spanish MINECO (MTM2014-57129-C2-1-P) and Junta de Castilla y León (VA057U16, VA137G18). Ş. Kuru acknowledges Ankara University and the warm hospitality at Department of Theoretical Physics, University of Valladolid, where part of this work has been done. L. Sourrouille acknowledges the partial support from CONICET to develop this work at the University of Valladolid.

\section{References}

[1] A. H. Castro Neto, F. Guinea, N. M. R. Peres, K. S. Novoselov and A. K. Geim, The electronic properties of graphene, Rev. Mod. Phys., 81, 109 (2009).

[2] G. W. Semenoff, Condensed-Matter Simulation of a Three-Dimensional Anomaly, Phys. Rev. Lett., 53, 2449 (1984).

[3] A. De Martino, L. Dell Anna and R. Egger, Magnetic Confinement of massless Dirac fermions in graphene, Phys. Rev. Lett., 98, 066802 (2007).

[4] M. Ramezani Masir, A. Matulis and F. M. Peeters, Quasibound states of Schrödinger and Dirac elèctrons in a magnetic quantum dot, Phys. Rev. B, 79, 155451 (2009).

[5] C. A. Downing and M. E. Portnoi, Massless Dirac fermions in two dimensions: Confinement in nonuniform magnetic fields, Phys. Rev. B, 94, 165407 (2016). 
[6] C. A. Downing and M. E. Portnoi, Magnetic quantum dots and rings in two dimensions, Phys. Rev. B, 94, 045430 (2016).

[7] M. I. Katsnelson, K. S. Novoselov and A. K. Geim, Chiral tunnelling and the Klein paradox in graphene, Nat. Phys., 2, 620 (2006).

[8] C.-L. Ho and P. Roy, On zero energy states in graphene, EPL, 108, 20004 (2014).

[9] A. V. Rozhkov, G. Giavaras, Y. P. Bliokh, V. Freilikher and F. Nori, Electronic properties of mesoscopic graphene structures: Charge confinement and control of spin and charge transport, Phys. Rep., 503, 77 (2011).

[10] D. A. Stone, C. A. Downing and M. E. Portnoi, Searching for confined modes in graphene channels: The variable phase method, Phys. Rev. B, 86, 075464 (2012).

[11] S. J. Lee, S. Souma, G. Ihm and K. J. Chang, Magnetic quantum dots and magnetic edge states, Phys. Rep., 394, 1 (2004).

[12] A. Nogaret, Electron dynamics in inhomogeneous magnetic fields, J. Phys.: Condens. Matter, 22, 253201 (2010).

[13] S. Park and H.-S. Sim, Magnetic edge states in graphene in nonuniform magnetic fields, Phys. Rev. B, 77, 075433 (2008).

[14] A. Kormanyos, P. Rakyta, L. Oroszlany and J. Cserti, Bound states in inhomogeneous magnetic field in graphene: Semiclassical approach, Phys. Rev. B, 78, 045430 (2008).

[15] S. Ghosh and M. Sharma, Electron optics with magnetic vector potential barriers in graphene, J. Phys.: Condens. Matter, 21, 292204 (2009).

[16] S. Kuru, J. Negro and L. M. Nieto, Exact analytic solutions for a Dirac electron moving in graphene under magnetic fields, J. Phys.: Condens. Matter, 21, 455305 (2009).

[17] T. K. Ghosh, Exact solutions for a Dirac electron in an exponentially decaying magnetic field, J. Phys.: Condens. Matter, 21, 045505 (2008).

[18] L. Z. Tan, C.-H. Park and S. G. Louie, Graphene Dirac fermions in one-dimensional inhomogeneous field profiles: Transforming magnetic to electric field, Phys. Rev. B, 81, 195426 (2010).

[19] E. Milpas, M. Torres and G. Murguia, Magnetic field barriers in graphene: an analytically solvable model, J. Phys.: Condens. Matter, 23, 245304 (2011).

[20] M. Ramezani Masir, P. Vasilopoulos and F. M. Peeters, Graphene in inhomogeneous magnetic fields: bound, quasi-bound and scattering states, J. Phys.: Condens. Matter, 23, 315301 (2011). 
[21] D. Wang and G. Jin, Magnetically confined states of Dirac electrons in a graphenebased quantum annulus, Europhys. Lett., 88, 17011 (2009).

[22] C. M. Lee, R. C. H. Lee, W. Y. Ruan and M. Y. Chou, Low-lying spectra of massless Dirac electron in magnetic dot and ring, Appl. Phys. Lett., 96, 212101(2010).

[23] N. Lindvall, A. Shivayogimatg and A. Yurgens, Measurements of weak localization of graphene in inhomogeneous magnetic fields, JETP Letters, 102, 367-371 (2015).

[24] J. Güttinger, C. Stampfer, T. Frey, T. Ihn and K. Ensslin, Graphene quantum dots in perpendicular magnetic fields, Phys. Status Solidi B, 246, 25532557 (2009).

[25] K. S. Novoselov, Z. Jiang, Y. Zhang, S. V. Morozov, H. L. Stormer, U. Zeitler, J. C. Maan, G. S. Boebinger, P. Kim and A. K. Geim, Room-temperature quantum Hall effect in graphene, Science, 315, 1379 (2007).

[26] A. Concha and Z. Tesanovic, Effect of a velocity barrier on the ballistic transport of Dirac fermions, Phys. Rev. B, 82, 033413 (2010).

[27] C. A. Downing and M. E. Portnoi, Localization of massless Dirac particles via spatial modulations of Fermi velocity, J. Phys.: Condens. Matter, 29, 315301 (2017).

[28] Y. Aharonov and A. Casher, Ground state of a spin- $1 / 2$ charged particle in a twodimensional magnetic field, Phys. Rev. A, 19, 2461-2462 (1979).

[29] M. I. Katsnelson and M. F. Prokhorova, Zero-energy states in corrugated bilayer graphene, Phys. Rev. B, 77, 205424 (2008).

[30] J. Kailasvuori, Pedestrian index theorem a la Aharonov-Casher for bulk threshold modes in corrugated multilayer graphene, EPL, 87, 47008 (2009).

[31] V. Jakubsky, Ş. Kuru, J. Negro and S. Tristao, Supersymmetry in spherical molecules and fullerenes under perpendicular magnetic fields, J. Phys.: Condens. Matter, 25, 165301 (2013).

[32] V. Jakubsky, S. Kuru and J. Negro, Carbon nanotubes in an inhomogeneous transverse magnetic field: exactly solvable model, J. Phys. A: Math. Theor., 47, 115307 (2014).

[33] F. Cooper, A. Khare and U. Sukhatme, Supersymmetry and Quantum Mechanics, Phys. Rep., 251, 267-385 (1995).

[34] G. Junker, Supersymmetric Methods in Quantum and Statistical Physics. Springer, Heidelberg (1996).

[35] B. Mielnik and O. Rosas-Ortiz, Factorization, little or great algorithm?, J. Phys. A: Math. Gen., 37, 10007-10035 (2004). 
[36] D. J. Fernandez C., Supersymmetric Quantum Mechanics, AIP Conf. Proc., 1287, 3-36 (2010).

[37] P. Roy, T. K. Ghosh and K. Bhattacharya, Localization of Dirac-like excitations in graphene in the presence of smooth inhomogeneous magnetic fields, J. Phys.: Condens. Matter, 24, 055301 (2012).

[38] M. R. Setare and D. Jahani, Quantum Hall effect and the different zero energy modes of graphene, Chinese Phys. Lett., 28, 097302 (2011). 